\title{
NOB1 silencing inhibits the growth and metastasis of laryngeal cancer cells through the regulation of JNK signaling pathway
}

\author{
XIN GAO, JIN WANG, WEILIANG BAI, WENYUE JI and LIPING WANG \\ Department of Otorhinolaryngology-Head and Neck Surgery, Shengjing Hospital of China Medical University, \\ Heping, Shenyang, Liaoning 110004, P.R. China
}

Received December 3, 2015; Accepted January 5, 2016

DOI: $10.3892 /$ or.2016.4707

\begin{abstract}
Nin one binding protein (NOB1) plays important roles in the synthesis and degradation of proteins, thus having effects on the cellular process. In the present study, the expression level of NOB1 in laryngeal cancer patients was detected by quantitative PCR and western blotting, and the effect of NOB1 on growth and metastasis of laryngeal cancer cells was explored. Silence of NOB1 was found to inhibit the proliferation of laryngeal cancer cells, arrest cell cycle and induce cell apoptosis. NOB1 silence was also found to inhibit the migration and invasion of laryngeal cancer cells and to downregulate the protein levels of matrix metalloproteinases (MMPs)-2 and MMP-9. Further mechanism study revealed that the JNK signaling pathway was involved in the function of NOB1. Our present results suggest that NOB1 plays an oncogenic role in laryngeal cancer cells through the regulation of JNK signaling pathway, and lays a theoretical foundation for further exploration of NOB1.
\end{abstract}

\section{Introduction}

Laryngeal cancer is a common malignant tumor appearing in head and neck. More than $90 \%$ of laryngeal cancer is laryngeal squamous cell carcinoma which accounts for $14 \%$ of the squamous cell carcinoma of head and neck $(1,2)$. Despite advances in therapies, the incidence of laryngeal cancer is high with $\sim 160,000$ new cases each year and the mortality of laryngeal cancer is still high with a 64\% 5-year survival rate (3). Tumorigenesis of laryngeal cancer is a complex process involved in genetic dysregulation. Understanding fully the underlying mechanism of laryngeal cancer tumorigenesis is imperative to the therapy of laryngeal cancer.

Nin one binding protein (NOB1) gene is located on human chromosome 16q22.1 (4) and NOB1 protein is mainly

Correspondence to: Professor Liping Wang, Department of Otorhinolaryngology-Head and Neck Surgery, Shengjing Hospital of China Medical University, 36 Sanhao Street, Heping, Shenyang, Liaoning 110004, P.R. China

E-mail: wanglp@sj-hospital.org

Key words: Nin one binding protein, proliferation, cell cycle, apoptosis, migration, invasion, laryngeal cancer, JNK expressed in liver, lung and spleen. NOB1 protein is a nuclear protein composed of the PIN domain and the $\mathrm{C}$ terminal zinc ribbon domain. NOB1 regulates the maturation process of $18 \mathrm{~S}$ rRNA through the PIN domain, and influences the formation of ribosome, thus having impact on the synthesis of proteins (5-7). NOB1 also promotes the maturation of $20 \mathrm{~S}$ proteasome as well as the formation of $26 \mathrm{~S}$ proteasome, mediating the ubiquitin-mediated protein degradation $(8,9)$. NOB1 plays crucial roles in the synthesis and degradation of proteins, which indicates the importance of NOB1 in multiple physiologic activities of cells.

Recent studies show that NOB1 has higher expression in various tumors, such as breast (10), prostate (11) and lung cancer (12), than the adjacent non-tumor tissues. Inhibition of NOB1 is shown to perform anticancer function in breast (13), prostate (11), colon (14) and ovarian cancer (15). However, no data show the expression and function of NOB1 in laryngeal cancer. In the present study, we detected the expression level of NOB1 in laryngeal cancer patients and explored the effect of NOB1 silence on the proliferation, apoptosis and migration of laryngeal cancer cells. Results of the present study showed that NOB1 acted as an oncogene in laryngeal cancer cells and silence of NOB1 may become a promising therapeutic method in the treatment of laryngeal cancer.

\section{Materials and methods}

Clinical exploration. Paired laryngeal cancers and adjacent normal tissues were obtained from 28 patients who underwent primary surgical resection of laryngeal cancer in Shengjing Hospital of China Medical University from April, 2014 to April, 2015. Following surgical removal, these tissue samples were cut into small pieces immediately and frozen in liquid nitrogen. These tissue samples were subjected to quantitative real-time PCR (RT-qPCR) and western blotting as described below. The relative mRNA level of NOB1 in laryngeal cancer patients was calculated using the $2^{-\Delta \Delta \mathrm{Ct}}$ method and the relative protein level of NOB1 was normalized to $\beta$-actin. The study protocol was approved by the Human Research Ethics Committee of China Medical University.

Cell culture. Human laryngeal cancer cell line Hep2 was obtained from Type Culture Collection Center of Chinese Academy of Science (Shanghai, China). Cells were cultured in 
Table I. Sequence of shRNA.

Sense $\left(5^{\prime}->3^{\prime}\right)$

Antisense (5'->3')

\begin{tabular}{lll}
\hline NOB1 shRNA & GATCCCCGTGAGGACGTTCCAAGTGATTCA & AGCTAAAAAGTGAGGACGTTCCAAGTGATC \\
& AGAGATCACTTGGAACGTCCTCACTTTTT & TCTTGAATCACTTGGAACGTCCTCACGGG \\
NC & GATCCCCTTCTCCGAACGTGTCACGTTTCAA & AGCTAAAAATTCTCCGAACGTGTCACGTTCT \\
& GAGAACGTGACACGTTCGGAGAATTTTT & CTTGAAACGTGACACGTTCGGAGAAGGG
\end{tabular}

NOB1, Nin one binding protein; NC, negative control.

Table II. Sequence of primers used in RT-qPCR.

Forward primer $\left(5^{\prime}->3^{\prime}\right)$

Reverse primer (5'->3')

\begin{tabular}{lll}
\hline NOB1 & GCTTGTGAGCCTGAGAACCTG & TTATCCAGCCACCCCCGTC \\
$\beta$-actin & CTTAGTTGCGTTACACCCTTTCTTG & CTGTCACCTTCACCGTTCCAGTTT
\end{tabular}

NOB1, Nin one binding protein.

RPMI-1640 medium (Gibco, Grand Island, NY, USA) supplemented with $10 \%$ fetal bovine serum (FBS; HyClone, Logan, UT, USA) and maintained in a humid atmosphere at $37^{\circ} \mathrm{C}$ with $5 \% \mathrm{CO}_{2}$.

Infection. Sequence containing NOB1 shRNA or its corresponding negative control (NC) (Table I) was synthesized by Sangon Biotech (Shanghai, China) and inserted into pRNA-H1.1 plasmid. After sequencing confirmation, these two plasmids were named as NOB1 shRNA and NC, respectively. Cells were seeded into 6-well plates and infected with NOB1 shRNA or NC using Lipofectamine 2000 reagent (Invitrogen, Carlsbad, CA, USA) according to the manufacturer's protocol. Then, cells were cultured in RPMI-1640 medium supplemented with $10 \% \mathrm{FBS}$ and $100 \mu \mathrm{g} / \mathrm{ml} \mathrm{G} 418$ (Invitrogen) for positive colony selection.

MTT assay. Cells were seeded into 96-well plates $\left(3 \times 10^{3}\right.$ cells/well $)$ in quintuplicate, and MTT was added into each well at $0,24,48,72$ and $96 \mathrm{~h}$. After incubation for additional $4 \mathrm{~h}$, the supernatant was removed gently and $200 \mu \mathrm{l}$ dimethyl sulfoxide (DMSO) was added into each well. The absorbance at $490 \mathrm{~nm}$ was measured using a microplate reader.

Colony formation. Cells were seeded into cell culture dishes (500 cells/dish). The dishes were maintained at $37^{\circ} \mathrm{C}$ for 7 days to allow the colonies to form. The culture medium was changed every 2-3 days. After the colonies were formed, they were washed with phosphate-buffered saline (PBS), fixed with $4 \%$ paraformaldehyde and stained with Wright-Giemsa dye (Jiancheng Bio, Nanjing, China) for $5 \mathrm{~min}$. Images of the stained colonies were captured and the ratio of colony formation ( $>50$ cells/colony) was calculated.

Cell cycle assay. The cell cycle assay was performed by flow cytometry. Cells were harvested, washed with PBS and fixed with ice-cold $70 \%$ ethanol. The fixed cells were stained with Cell Cycle Detection kit (Beyotime, Shanghai, China), and incubated at $37^{\circ} \mathrm{C}$ for $30 \mathrm{~min}$ in the dark. Then, the cell cycle distribution was analyzed by flow cytometer (Becton-Dickinson, Franklin Lakes, NJ, USA).

Apoptosis assay. The apoptosis of cells in each group was evaluated by flow cytometry using a Cell Apoptosis Detection kit (Wanleibio, Shenyang, China). Cells were harvested and resuspended in $500 \mu \mathrm{l}$ binding buffer. Then, $5 \mu \mathrm{l}$ Annexin V-FITC and $5 \mu \mathrm{l}$ propidium iodide was added into the suspension. The cell suspension was then incubated at room temperature for $15 \mathrm{~min}$ in the dark. Then, apoptosis level of cells in each group was detected using flow cytometry.

Hoechst staining. For Hoechst staining, cells were seeded in a 12 -well plate ( $1 \times 10^{5}$ cells/well). Cells were fixed after culture at $37^{\circ} \mathrm{C}$ for $24 \mathrm{~h}$. After washing with PBS, cells were stained with Hoechst staining kit (Beyotime), then cells were observed under fluorescence microscope with a magnification of $x 400$, and the images were captured.

Wound-healing assay. Cells were seeded into 6-well plates. When cell confluence was $80-90 \%$, cells were treated with $1 \mu \mathrm{g} / \mathrm{ml}$ mitomycin $\mathrm{C}$ for $1 \mathrm{~h}$, and then $200 \mu \mathrm{l}$ pipette tips were used to make scratches on the cell monolayer to generate a wound. After the scratches were made, cells were cultured in serum-free medium and images of the wounds were captured at 0,24 and $48 \mathrm{~h}$. The migration rate was calculated by measuring the gap size of the wounds. Migration rate $=(1-$ gap size at 24 or $48 \mathrm{~h} /$ gap size at $0 \mathrm{~h}$ ) $\mathrm{x} 100 \%$.

Transwell assay. After infection with NOB1 shRNA, cells were made into cell suspension and $200 \mu \mathrm{l}$ cell suspension of each group was added into the upper chamber of Transwell plate (Corning, Tewksbury, MA, USA) pre-coated with 

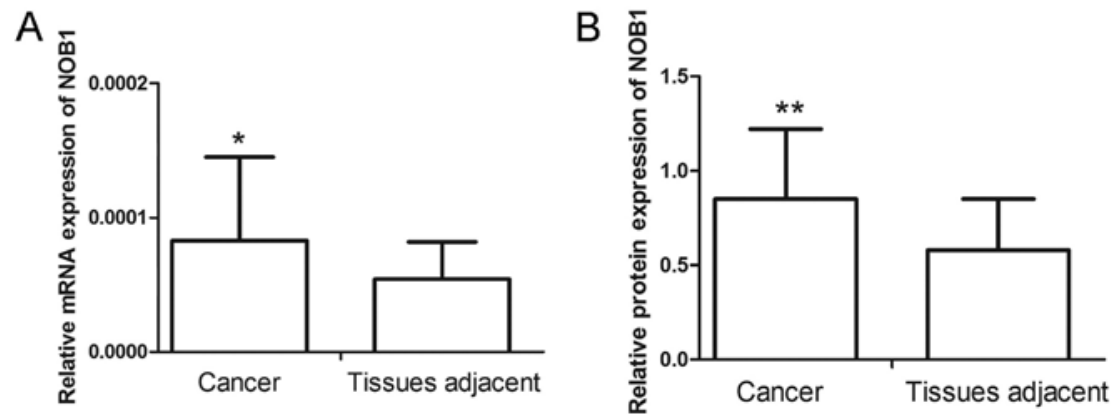

Figure 1. NOB1 level in laryngeal cancer patients. Paired laryngeal cancers and adjacent normal tissues were collected and the level of NOB1 was measured using (A) RT-qPCR and (B) western blotting. The results are presented as mean $\pm \mathrm{SD} ;{ }^{*} \mathrm{p}<0.05,{ }^{* *} \mathrm{p}<0.01$.

Matrigel (Becton-Dickinson) at a density of $2 \times 10^{4}$ cells/well. Medium $(800 \mu 1)$ with $20 \%$ FBS were added into the lower chambers. After incubation at $37^{\circ} \mathrm{C}$ for $24 \mathrm{~h}$, cells above the microporous membrane were removed by cotton swabs and cells at the bottom of the microporous membrane were fixed with $4 \%$ paraformaldehyde for $20 \mathrm{~min}$ at room temperature, and stained with $0.5 \%$ crystal violet for $5 \mathrm{~min}$. Images of cells in five random fields were captured using light microscopy with a magnification of $\mathrm{x} 200$.

$R T-q P C R$. Total RNA was extracted using total RNA extraction kit (BioTeke, Beijing, China) according to the manufacturer's protocol and reverse transcribed to cDNA with super M-MLV reverse transcriptase (BioTeke) and oligo(dT) $)_{15}$. The mRNA level of NOB1 was detected by SYBR-Green quantitative real-time PCR with primers as in Table II. The relative mRNA level of NOB1 was calculated using the $2^{-\Delta \Delta C \mathrm{Ct}}$ method (16).

Western blotting. Cells were harvested and lysed in RIPA lysis buffer (Beyotime) with 1\% phenylmethanesulfonyl fluoride, and protein was extracted by centrifugation. After measurement of protein concentration with a BCA protein assay kit (Beyotime), equal amount of protein from each group was injected into SDS-PAGE for electrophoresis. Then, protein was transferred to polyvinylidene fluoride (PVDF) membranes. After blockade with 5\% skim milk or 1\% BSA, the membranes were incubated with the primary antibodies against NOB1 (1:1,000; Proteintech, Chicago, IL, USA), cleaved-caspase-3, cleaved-PARP (1:1,000; Abcam, Cambridge, UK), matrix metalloproteinases (MMPs)-2, MMP-9, B-cell lymphoma-2 (Bcl-2), Bcl-2-associated X protein (Bax) (1:400; Boster, Wuhan, China), p-JNK, JNK (1:500; Bioss, Beijing, China) and $\beta$-actin (1:1,000; Santa Cruz Biotechnology, Inc., Dallas, TX, USA). After washing with TBST, the membranes were incubated with corresponding horseradish peroxidase-conjugated secondary antibodies (1:5,000; Beyotime). Then, the membranes were visualized using an enhanced chemiluminescence (ECL) detection system and the gray scale analysis was carried out with Gel-Pro-Analyzer.

Statistical analysis. All experiments were repeated three times and the results are presented as means \pm standard deviation (SD). One-way analysis of variance (ANOVA) and Bonferroni's multiple comparison were used to analyze the difference between each group. $p<0.05$ was considered to be significant.
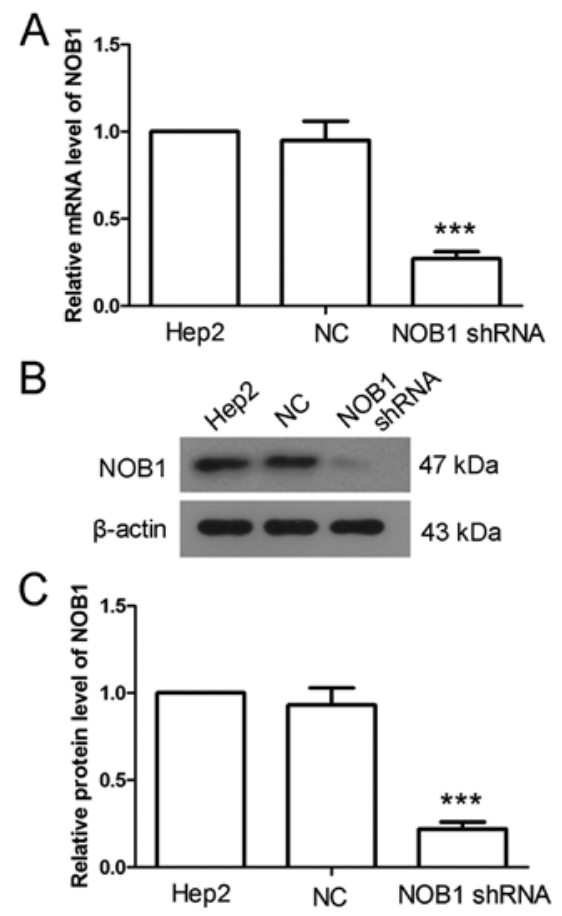

Figure 2. NOB1 shRNA downregulates the NOB1 level in laryngeal cancer cells. (A) After infection with NOB1 shRNA, the mRNA level of NOB1 was measured by RT-qPCR. (B and C) The protein level of NOB1 was detected by western blotting after infection with NOB1 shRNA. $\beta$-actin was used as the internal reference. Each experiment was repeated three times. Typical results are presented. The results are presented as mean $\pm \mathrm{SD} ;{ }^{* * *} \mathrm{p}<0.001$.

\section{Results}

NOB1 level in laryngeal cancer patients. The NOB1 level in laryngeal cancer patients was detected by RT-qPCR and western blotting. Results of RT-qPCR showed that laryngeal cancer tissues had a higher NOB1 level than those of the adjacent tissues (Fig. 1A). Similar to the results of RT-qPCR, western blotting also showed that NOB1 had a higher level in laryngeal cancer (Fig. 1B). These results demonstrated that NOB1 expressed at high level in laryngeal cancer.

NOB1 shRNA decreases the level of NOB1. NOB1 shRNA was used to explore the function of NOB1. RT-qPCR and western blotting were employed to detect the effect of NOB1 shRNA. Results of RT-qPCR showed that, in cells infected with NOB1 shRNA, the NOB1 level was decreased to $27 \pm 4 \%$ (Fig. 2A), 

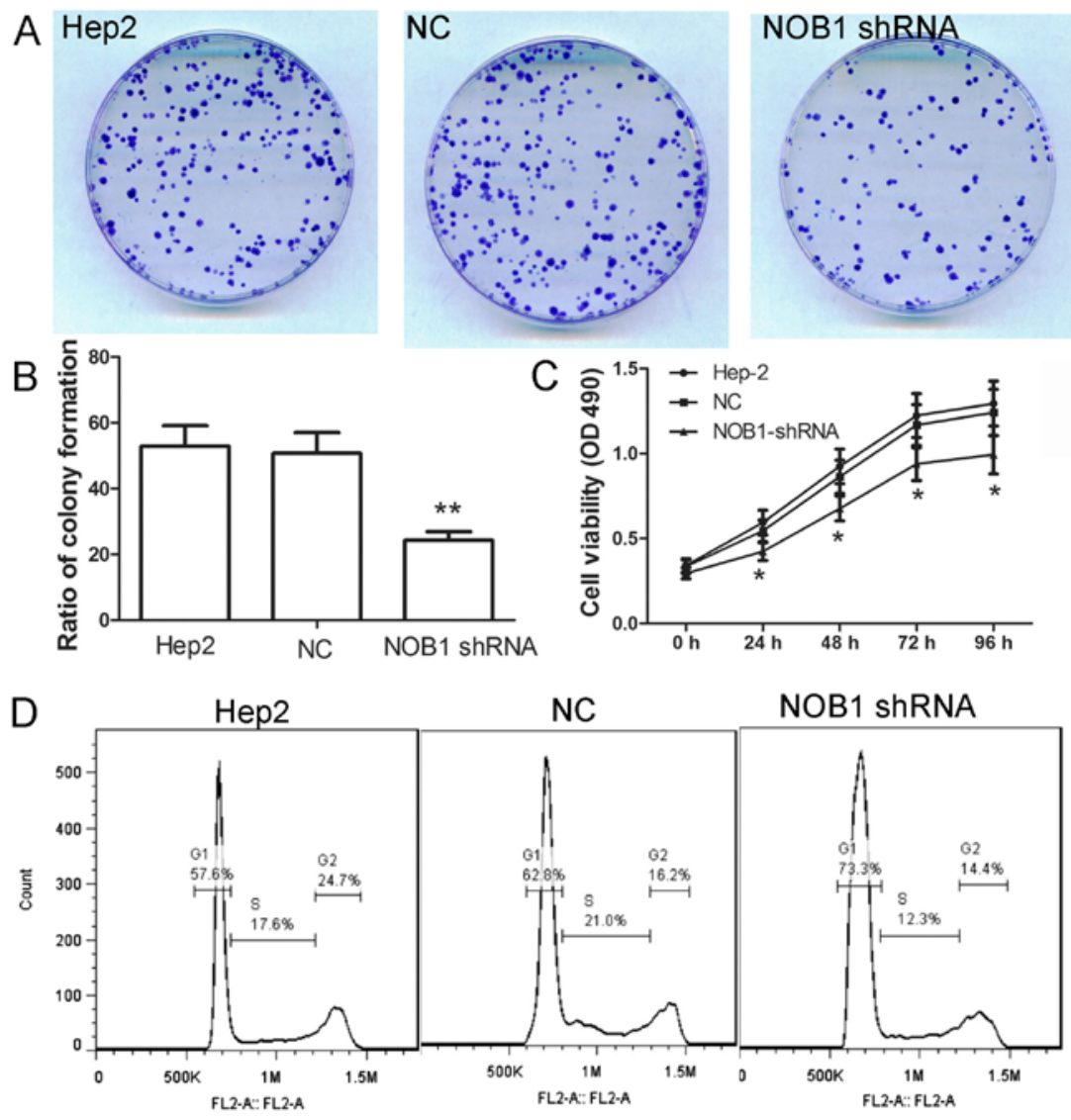

E

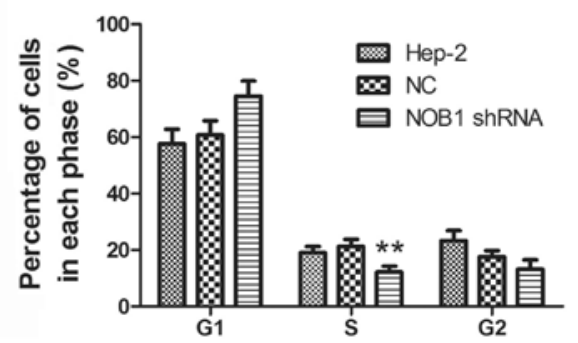

Figure 3. NOB1 shRNA inhibits the growth of laryngeal cancer cells. (A and B) Colony formation assay was performed after infection with NOB1 shRNA and the ratio of colony formation was calculated. (C) After infection with NOB1 shRNA, the cell viability was measured using MTT. (D and E) Cell cycle assay was performed after infection with NOB1 shRNA and the distribution of cell cycle was analyzed. All experiments were repeated three times and typical results are presented. Results are presented as mean $\pm \mathrm{SD} ;{ }^{*} \mathrm{p}<0.05,{ }^{* *} \mathrm{p}<0.01$.

but there was no significant change in cells infected with NC. Similar altered patterns were also found in the results of western blotting, after infected with NOB1 shRNA, the protein level of NOB1 was decreased to $22 \pm 4 \%$ (Fig. 2B and C). These results demonstrate that NOB1 shRNA downregulates NOB1 level effectively.

NOB1 shRNA inhibits the proliferation of laryngeal cancer cells. To explore the effect of NOB1 on the proliferation of laryngeal cancer cells, colony formation assay was carried out to explore the effect of NOB1. After infection with NOB1 shRNA, the ratio of colony formation was significantly decreased comparing with that of cells infected with NC (Fig. 3A and B; p <0.01). Then, MTT assay was also carried out. As shown in Fig. 3C, cells infected with NOB1 shRNA showed a slow growth comparing to cells infected with NC. These results suggest that NOB1 shRNA inhibits growth of laryngeal cancer cells.
Cell cycle is a crucial event that influences the growth of cells. The effect of NOB1 shRNA on cell cycle was detected in the present study. As shown in Fig. 3D and E, after infection with NOB1 shRNA, the percentage of cells in G1 phase was increased from $60.67 \pm 5.14$ to $74.47 \pm 5.44 \%$, and the percentage of cells in S phase was decreased from $21.30 \pm 2.46$ to $12.23 \pm 3.36 \%$. The distribution of cell cycle was changed after infection with NOB1 shRNA, which indicates the important role of NOB1 in the cell cycle.

NOB1 shRNA induces laryngeal cancer cell apoptosis. Cell apoptosis is also an important event that influences cell growth. Effect of NOB1 shRNA on cell apoptosis was detected using flow cytometry. Results showed that the percentage of apoptotic cells was $4.12 \pm 0.54 \%$ in cells infected with $\mathrm{NC}$, but the percentage of apoptosis was increased to $22.25 \pm 2.46 \%$ after infection with NOB1 shRNA (Fig. 4A and B). Hoechst staining was also employed 

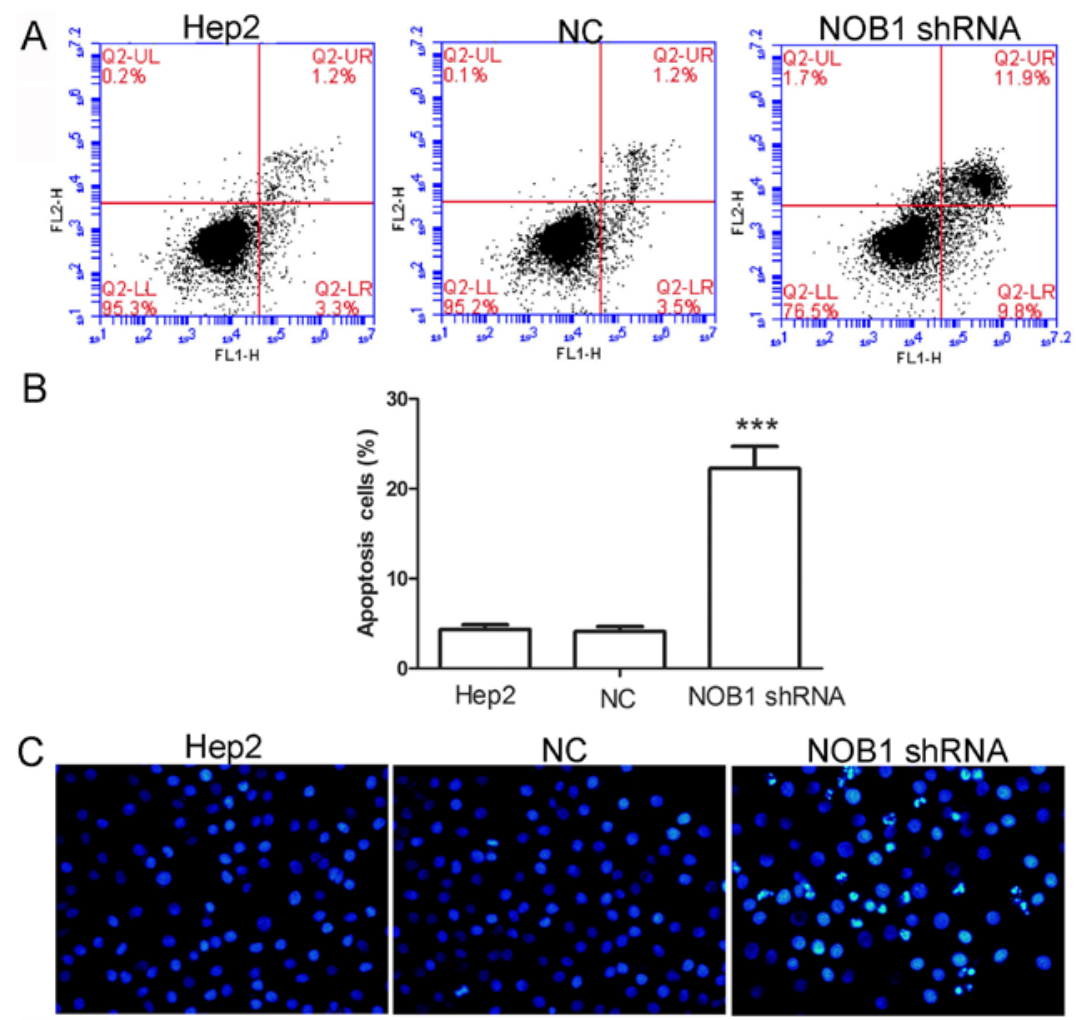

NOB1 shRNA
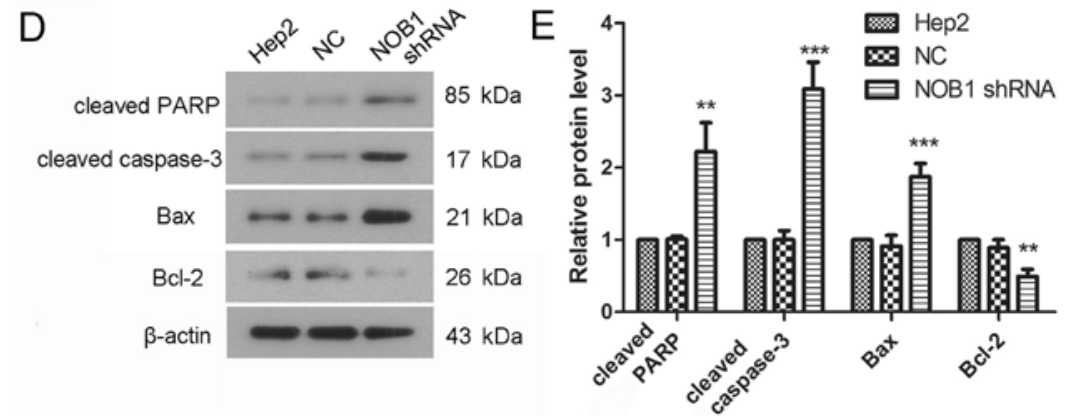

Figure 4. NOB1 silencing induces cell apoptosis. (A and B) Cell apoptosis was detected using flow cytometry and the percentage of cells in each phase was calculated. (C) Hoechst staining was used to detect cell apoptosis after infection with NOB1 shRNA. (D and E) Protein levels of cleaved PARP, cleaved caspase-3, Bax and Bcl-2 were detected using western blotting with $\beta$-actin as the internal reference. All experiments were repeated three times. Typical results are presented and results are presented as mean $\pm \mathrm{SD} ;{ }^{* *} \mathrm{p}<0.01,{ }^{* * *} \mathrm{p}<0.001$.

to detect cell apoptosis. As shown in Fig. 4C, there was obvious pyknosis of chromatin in cells infected with NOB1 shRNA. The protein levels of cleaved caspase-3, cleaved PARP, Bcl-2 and Bax were also detected in the present study. Results of western blotting showed that the protein level of cleaved caspase-3 was increased to 3.09 \pm 0.37 -fold, the protein level of cleaved PARP was increased to $2.2 \pm 0.4$-fold, the protein level of Bax was increased to $1.87 \pm 0.19$-fold, but the protein level of Bcl-2 was decreased to $49 \pm 10 \%$ (Fig. 4D and E). These results demonstrate that NOB1 shRNA induces apoptosis of laryngeal cancer cells.

NOB1 shRNA inhibits migration and invasion of laryngeal cancer cells. The effect of NOB1 shRNA on migration of laryngeal cancer cells was also explored in the present study. Wound-healing and Transwell assays were performed to detect the cell migration capability. Results of wound-healing assay showed that the migration capability of cells infected with NOB1 shRNA was significantly decreased at both 24 and
$48 \mathrm{~h}$ (Fig. 5). In the results of Transwell assay, the number of cells passing through the micropore membrane in the NOB1 shRNA group was 72.2 \pm 7.46 , significantly lower than of the NC group (119.4 \pm 12.9 ) (Fig. 6A). MMP-2 and MMP-9 play important roles in the process of cell migration and were detected by western blotting in the present study. The protein levels of MMP-2 and MMP-9 were decreased to $64 \pm 7$ and $52 \pm 7 \%$, respectively, in cells infected with NOB1 shRNA, significantly lower than that of cells infected with NC (Fig. 6B and C). These results suggest that downregulation of NOB1 inhibits the migration of laryngeal cancer cells.

JNK signaling pathway is involved in the function of NOB1. JNK and P38 signaling pathways play important roles in various events, such as cell growth and migration. In the present study, the protein levels JNK, P38, phosphorylated JNK (p-JNK) and phosphorylated P38 (p-P38) were detected by western blotting. As shown in Fig. 7, there was a significant increase in the JNK phosphorylation level after infection 


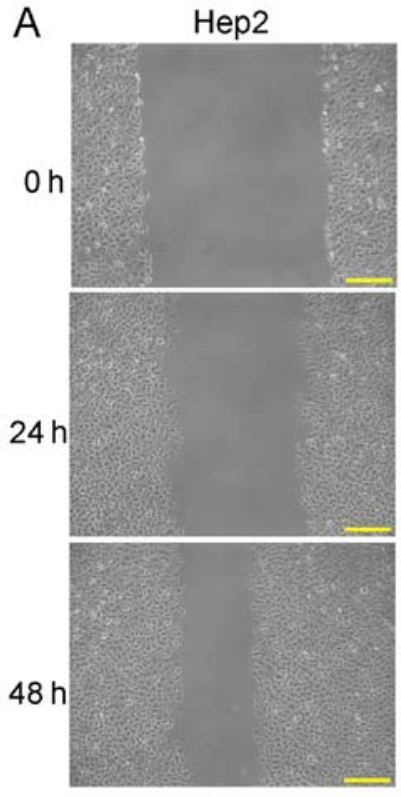

B

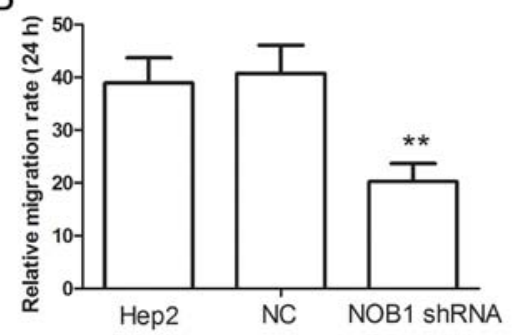

NC
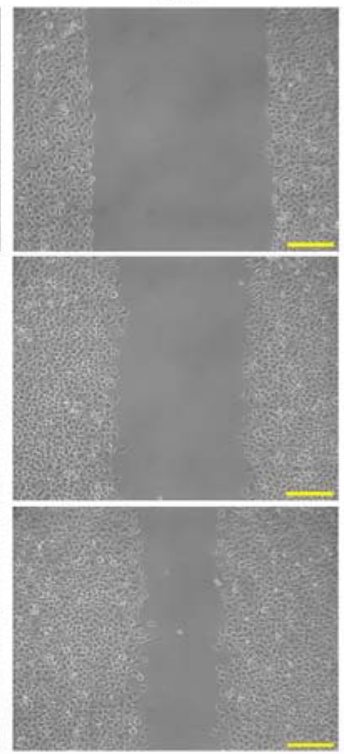

C



Figure 5. Silencing of NOB1 inhibits migration of laryngeal cancer cells. (A) After infection with NOB1 shRNA, wound-healing assay was performed to detect changes in migration capacity. (B) Relative migration rate at $24 \mathrm{~h}$. (C) Relative migration rate at $48 \mathrm{~h}$. Each experiment was repeated three times and typical results are presented. Results are shown as mean $\pm \mathrm{SD} ;{ }^{* *} \mathrm{p}<0.01$.

A

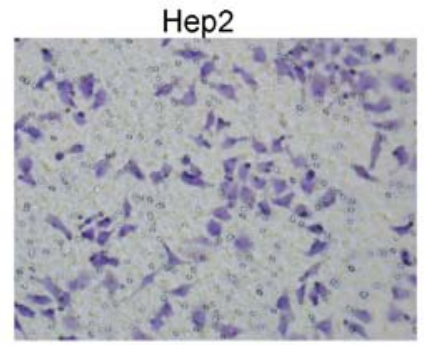

NOB1 SRNA

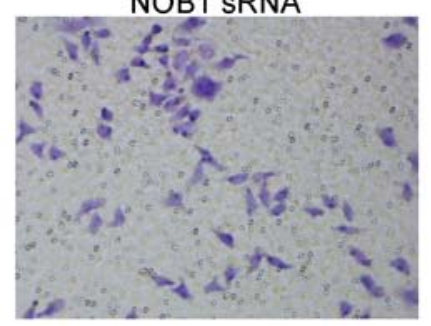

B

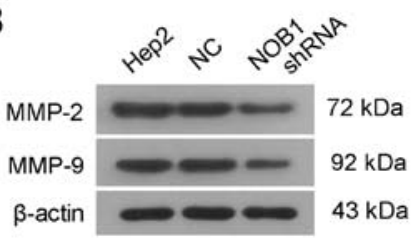

NC
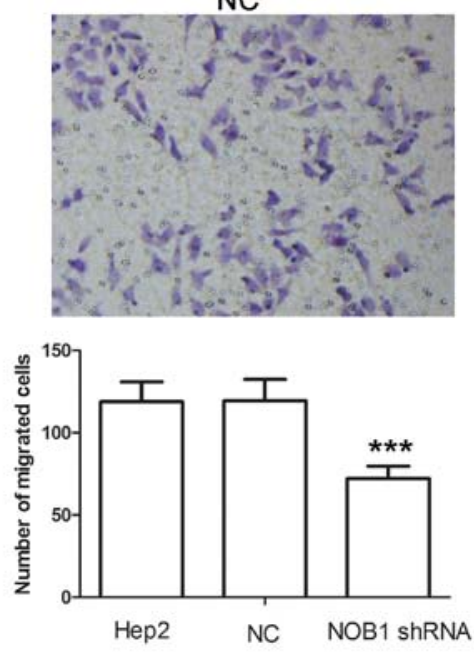

C

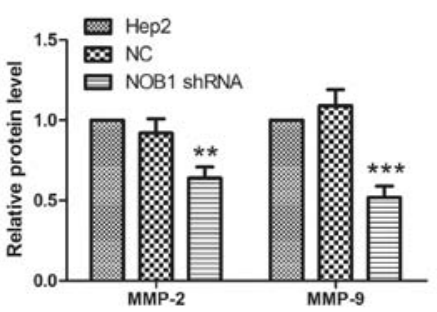

Figure 6. Silencing of NOB1 inhibits invasion of laryngeal cancer cells. (A) Transwell assay was carried out after infection with NOB1 shRNA and the number of cells passing through the micropore membrane was calculated. (B and C) Protein levels of MMP-2 and MMP-9 were detected after infection with NOB1 shRNA. All experiments were repeated three times. Results are presented as mean $\pm \mathrm{SD}$ and typical results are presented; ${ }^{* *} \mathrm{p}<0.01,{ }^{* * *} \mathrm{p}<0.001$. 
A

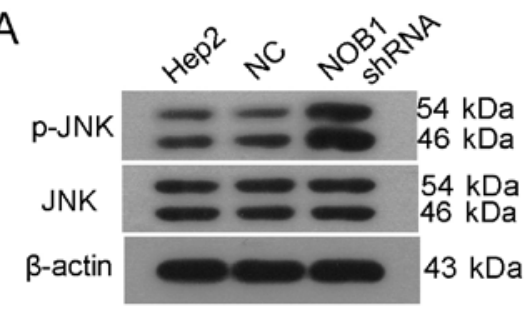

C

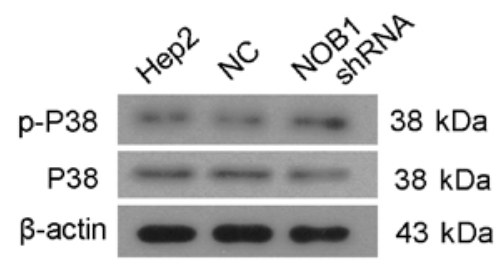

B

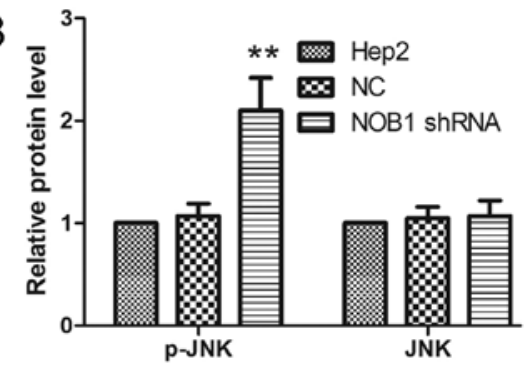

D



Figure 7. NOB1 shRNA induces the activation of JNK. (A and B) Protein levels of JNK and phosphorylated JNK (p-JNK) were detected by western blotting. $(\mathrm{C}$ and $\mathrm{D})$ Western blotting was used to detect the protein level of P38 and phosphorylated P38 (p-P38). Each experiment was repeated three times and the results are presented as mean $\pm \mathrm{SD}$. Typical results are presented; ${ }^{* *} \mathrm{p}<0.01$.

with NOB1 shRNA, leaving no significant changes in protein level of JNK (Fig. 7A and B; p<0.01). After infection with NOB1 shRNA, the P38 phosphorylation level showed a slight increase, but not significantly (Fig. 7C and D). These results indicate that JNK signaling pathway may be involved in function of NOB1.

\section{Discussion}

In the present study, we found the expression level of NOB1 in laryngeal cancer patients was high and we further explored the function of NOB1 on proliferation, apoptosis and migration of laryngeal cancer cells. Silence of NOB1 was found to inhibit the proliferation of laryngeal cancer cells, arrest cell cycle process and induce apoptosis. NOB1 silence also inhibited the migration and invasion of laryngeal cancer cells. Further mechanism study showed that the JNK was activated after infection with NOB1 shRNA, which indicates that the function of NOB1 on proliferation and migration of laryngeal cancer cells may be associated with JNK signaling pathway.

In the process of tumorigenesis, the expression of multiple genes has been shown out of control. NOB1 shows high level in breast (10) and prostate cancer (11), and is associate with these cancers $(11,13)$. In the present study, clinical detection showed that the expression level of NOB1 in laryngeal cancer was high. This indicates that NOB1 may be associated with tumorigenesis of laryngeal cancer.

Dysregulation of cell growth is a characteristic of cancer. In the present study, we found that silencing of NOB1 inhibited the growth and colony formation capability of laryngeal cancer cells. NOB1 was reported to influence the growth of breast (13), ovarian (15), colon (14), prostate (11) and renal cancer (17), which was consistent with the results of the present study. Cell cycle is an important event in the process of cell growth, and in the present study, NOB1 showed a regulatory role in the cell cycle process of laryngeal cancer cells. Knockdown of NOB1 was also reported to have influence on the expression of cell cycle-related genes, such as cyclin and CDKs (18), and induce cell cycle arrest at G0/G1 phase (11,13-15,17-19).

Apoptosis is also an important event that has influence on cell growth. Results of the present study showed that NOB1 had an anti-apoptosis function in laryngeal cancer cells. Consistent with the present study, downregulation of NOB1 was reported to induce cell apoptosis in colon (20) and lung cancer (21). The ratio of Bax and Bcl-2 is associated with the opening of mitochondrion permeability transition pore and the release of cytochrome $c$ which activates caspase-9 and induces cell apoptosis. In the present study, the expression of Bax and Bcl-2 was also found to be regulated by NOB1 silence. These results prompted us to clarify whether the effect of NOB1 silencing on apoptosis is associated with the mitochondria-mediated apoptosis. Cell apoptosis is an important way through which radiotherapy and chemotherapy kill tumor cells. Meng et al reported that silence of NOB1 enhanced the sensitivity of tumor cells to radiotherapy (22) and Liu et al also showed that silence of NOB1 boosted the anticancer activity of chemotherapeutics drugs (23). The effects of NOB1 silencing on the proliferation and apoptosis of cancer cells suggested that NOB1 may be a promising therapeutic target in the treatment of laryngeal cancer.

Metastasis is a crucial cause of tumor deterioration. The expression level of NOB1 was reported to be associated with the mortality of cancer cells (24). Downregulation of NOB1 inhibits the migration and invasion of glioma (19), prostate cancer (11) and osteosarcoma (25). Consistent with these reports, in the present study, silencing of NOB1 was found to inhibit the migration and invasion of laryngeal cancer cells. MMPs are crucial enzymes that degrade extracellular matrix and contribute to the migration and invasion of cells. Downregulation of MMP-2 and MMP-9 by NOB1 shRNA 
was also observed in the present study. Silence of NOB1 was reported to increase the level of E-cadherin (25), which suggests the potential regulatory role of NOB1 in EMT process.

JNK is a member of the mitogen-activated protein kinases (MAPKs) which play important roles in cell proliferation, migration and differentiation. JNK is activated by various stimuli leading to contradictory responses (26), JNK phosphorylates the anti-apoptosis $\mathrm{Bcl}-2$ to promote apoptosis of cells (27) or phosphorylates the pro-apoptotic BAD to inhibit apoptosis (28). In laryngeal cancer cells, JNK plays a pro-apoptotic role (29). In the present study, we found that the NOB1 silence induced the activation of JNK and this indicated that JNK signaling may be involved in the function of NOB1. The activation of P38 was also detected in the present study, but no significant change was found after infection with NOB1 shRNA. These results indicate that P38 signaling may be not involved in the function of NOB1, at least in laryngeal cancer cells. However, Che et al showed that the expression level of NOB1 was associated with the activation of P38, and NOB1 silencing inhibited the expression of $\mathrm{P} 38$ in prostate carcinoma (24).

In the present study, silence of NOB1 was found to inhibit the proliferation and migration of laryngeal cancer cells, arrest cell cycle and induce apoptosis, and these functions of NOB1 may be associated with the JNK signaling pathway. NOB1, which is associated with the proliferation, apoptosis and migration of laryngeal cancer cells, may be a promising therapeutic target in the treatment of laryngeal cancer, and it may also show potential as a clinical marker of laryngeal cancer.

\section{Acknowledgements}

The present study was supported by grants from the Natural Science Foundation of Liaoning Province (no. 20092135). Ethical approval was given by the Medical Ethics Committee of Shengjing Hospital of China Medical University with the reference no. 2014PS17K.

\section{References}

1. Morshed K, Polz-Dacewicz M, Szymański M and Polz D: Short-fragment PCR assay for highly sensitive broad-spectrum detection of human papillomaviruses in laryngeal squamous cell carcinoma and normal mucosa: Clinico-pathological evaluation. Eur Arch Otorhinolaryngol 265 (Suppl 1): S89-S96, 2008.

2. Jemal A, Siegel R, Ward E, Hao Y, Xu J and Thun MJ: Cancer statistics, 2009. CA Cancer J Clin 59: 225-249, 2009.

3. Ramroth H, Schoeps A, Rudolph E, Dyckhoff G, Plinkert P, Lippert B, Feist K, Delank KW, Scheuermann K, Baier G, et al: Factors predicting survival after diagnosis of laryngeal cancer. Oral Oncol 47: 1154-1158, 2011.

4. Zhang Y, Ni J, Zhou G, Yuan J, Ren W, Shan Y, Tang W, Yu L and Zhao S: Cloning, expression and characterization of the human NOB1 gene. Mol Biol Rep 32: 185-189, 2005.

5. Fatica A, Oeffinger M, Dlakić M and Tollervey D: Noblp is required for cleavage of the $3^{\prime}$ end of $18 \mathrm{~S}$ rRNA. Mol Cell Biol 23: 1798-1807, 2003.

6. Fatica A, Tollervey D and Dlakić M: PIN domain of Nob1p is required for D-site cleavage in $20 \mathrm{~S}$ pre-rRNA. RNA 10: 1698-1701, 2004.

7. Lamanna AC and Karbstein K: Nob1 binds the single-stranded cleavage site D at the 3'-end of $18 \mathrm{~S}$ rRNA with its PIN domain. Proc Natl Acad Sci USA 106: 14259-14264, 2009.

8. Tone Y and Toh-E A: Noblp is required for biogenesis of the 26S proteasome and degraded upon its maturation in Saccharomyces cerevisiae. Genes Dev 16: 3142-3157, 2002.
9. Veith T, Martin R, Wurm JP, Weis BL, Duchardt-Ferner E, Safferthal C, Hennig R, Mirus O, Bohnsack MT, Wöhnert J, et al: Structural and functional analysis of the archaeal endonuclease Nob1. Nucleic Acids Res 40: 3259-3274, 2012.

10. Li XY, Luo QF, Li J, Wei CK, Kong XJ, Zhang JF and Fang L: Clinical significance of NOB1 expression in breast infiltrating ductal carcinoma. Int J Clin Exp Pathol 6: 2137-2144, 2013.

11. Zhang X, Zhang D, Qu F, Hong Y, Cao J, Pan X, Li L, Huang Y, Huang $\mathrm{H}$, Yin L, et al: Knockdown of NOB1 expression inhibits the malignant transformation of human prostate cancer cells. Mol Cell Biochem 396: 1-8, 2014.

12. Liu K, Gu MM, Chen HL and You QS: NOB1 in non-small-cell lung cancer: Expression profile and clinical significance. Pathol Oncol Res 20: 461-466, 2014

13. Huang WY, Chen DH, Ning L and Wang LW: siRNA mediated silencing of NIN1/RPN12 binding protein 1 homolog inhibits proliferation and growth of breast cancer cells. Asian Pac J Cancer Prev 13: 1823-1827, 2012.

14. Liu Y, Huang H, Yuan B, Zhuang LY, Luo TP and Zhang Q: Lentivirus-mediated knockdown of NOB1 suppresses the proliferation of colon cancer cells. Z Gastroenterol 52: 429-435, 2014.

15. Lin Y, Peng S, Yu H, Teng H and Cui M: RNAi-mediated downregulation of $N O B 1$ suppresses the growth and colony-formation ability of human ovarian cancer cells. Med Oncol 29: 311-317, 2012.

16. Livak KJ and Schmittgen TD: Analysis of relative gene expression data using real-time quantitative PCR and the $2^{-\Delta \Delta C t}$ method. Methods 25: 402-408, 2001.

17. Jia JW, Liu AQ, Wang Y, Zhao F, Jiao LL and Tan J: Evaluation of NIN/RPN12 binding protein inhibits proliferation and growth in human renal cancer cells. Tumour Biol 36: 1803-1810, 2015.

18. Lu Z, Guo Q, Shi A, Xie F and Lu Q: Downregulation of NIN/RPN12 binding protein inhibit the growth of human hepatocellular carcinoma cells. Mol Biol Rep 39: 501-507, 2012.

19. Wang H, Li P and Zhao B: Knockdown of NOB1 expression by RNAi inhibits cellular proliferation and migration in human gliomas. Gene 528: 146-153, 2013.

20. He XW, Feng T, Yin QL, Jian YW and Liu T: NOB1 is essential for the survival of RKO colorectal cancer cells. World $\mathrm{J}$ Gastroenterol 21: 868-877, 2015.

21. Li Y, Ma C, Qian M, Wen Z, Jing H and Qian D: Downregulation of NOB1 suppresses the proliferation and tumor growth of non-small cell lung cancer in vitro and in vivo. Oncol Rep 31: 1271-1276, 2014.

22. Meng W, Wang PS, Liu J, Xue S, Wang GM, Meng XY and Chen G: Adenovirus-mediated siRNA targeting NOB1 inhibits tumor growth and enhances radiosensitivity of human papillary thyroid carcinoma in vitro and in vivo. Oncol Rep 32: 2411-2420, 2014.

23. Liu J, Dong BF, Wang PS, Ren PY, Xue S, Zhang XN, Han Z and Chen G: Silencing NOB1 enhances doxorubicin antitumor activity of the papillary thyroid carcinoma in vitro and in vivo. Oncol Rep 33: 1551-1559, 2015.

24. Che JP, Li W, Yan Y, Liu M, Wang GC, Li QY, Yang B, Yao XD and Zheng JH: Expression and clinical significance of the nin one binding protein and $\mathrm{p} 38$ MAPK in prostate carcinoma. Int J Clin Exp Pathol 6: 2300-2311, 2013.

25. Chen B, Liu J, Wu D, Qin Y, Peng C, Li C and Wang J: Gene silencing of NOBI by lentivirus suppresses growth and migration of human osteosarcoma cells. Mol Med Rep 9: 2173$2179,2014$.

26. Bode AM and Dong Z: The functional contrariety of JNK. Mol Carcinog 46: 591-598, 2007.

27. Maundrell K, Antonsson B, Magnenat E, Camps M, Muda M, Chabert C, Gillieron C, Boschert U, Vial-Knecht E, Martinou JC, et al: $\mathrm{Bcl}-2$ undergoes phosphorylation by $\mathrm{c}$-Jun $\mathrm{N}$-terminal kinase/stress-activated protein kinases in the presence of the constitutively active GTP-binding protein Rac1. J Biol Chem 272: 25238-25242, 1997.

28. Yu C, Minemoto Y, Zhang J, Liu J, Tang F, Bui TN, Xiang J and Lin A: JNK suppresses apoptosis via phosphorylation of the proapoptotic Bcl-2 family protein BAD. Mol Cell 13: 329-340, 2004.

29. Brahim S, Aroui S, Abid K and Kenani A: Involvement of C-jun $\mathrm{NH}_{2}$-terminal kinase and apoptosis induced factor in apoptosis induced by deglycosylated bleomycin in laryngeal carcinoma cells. Cell Biol Int 33: 964-970, 2009. 